\title{
On common quadratic Lyapunov functions for stable discrete-time LTI systems
}

\author{
Oliver Mason, \\ Hamilton Institute, \\ NUI Maynooth, \\ Maynooth, Co. Kildare, \\ Ireland. \\ (oliver.mason@may.ie) \\ Robert Shorten ${ }^{1}$, \\ Hamilton Institute, \\ NUI Maynooth, \\ Maynooth, Co. Kildare, \\ Ireland. \\ (robert.shorten@may.ie)
}

${ }^{1}$ corresponding author 


\title{
On common quadratic Lyapunov functions for stable discrete-time LTI systems
}

\author{
Oliver Mason ${ }^{\dagger} \quad$ Robert Shorten ${ }^{\ddagger}$ \\ Hamilton Institute Hamilton Institute \\ NUI Maynooth NUI Maynooth \\ Ireland Ireland
}

\begin{abstract}
This paper deals with the question of the existence of weak and strong common quadratic Lyapunov functions (CQLFs) for stable discrete-time linear timeinvariant (LTI) systems. The main result of the paper provides a simple characterisation of pairs of such systems for which a weak CQLF of a given form exists but for which no strong CQLF exists. An application of this result to second order discrete-time LTI systems is presented.
\end{abstract}

\section{Introduction and Notation}

In recent years, the area of systems theory and control has witnessed a considerable growth of interest in systems characterised by a combination of continuous dynamics and logicbased switching. Systems of this type are commonly referred to as switching systems in the scientific and engineering literature. A major issue in this area is the determination of easily verifiable and interpretable conditions that guarantee the stability of such systems. For

\footnotetext{
$\dagger$ oliver.mason@may.ie

${ }_{\ddagger}^{\ddagger}$ robert.shorten@may.ie
} 
an overview of some of the more recent approaches to this issue see (DeCarlo et al. 2000), (Branicky 1994), (Ye, Michel and Hou 1998), (Shorten and Narendra 2000), (Shorten and Narendra 1998), (Johansson and Rantzer 1998), (Shorten and O'Cairbre 2002), (O'Cairbre and Shorten 2001), (Narendra and Balakrishnan 1994b). In this context the problem of the existence or non-existence of a common quadratic Lyapunov function (CQLF) for a family of linear time-invariant (LTI) systems is of great importance. There is already a considerable body of literature dedicated to this question for both discrete-time and continuous-time systems (Boyd and Yang 1989), (Ando 2001), (Agrachev and Liberzon 2001), (Shorten and Narendra 1999), (Akar and Narendra 2001), (Gurvits 1995), (Ooba and Funahashi 1999), (Ooba and Funahashi 1997), (Mori, Mori and Kuroe 1998), (Mori, Mori and Kuroe 2001), (Narendra and Balakrishnan 1994a). The main result presented here is concerned with the CQLF existence problem for a family of two discrete-time LTI systems.

Throughout $\mathbb{R}$ and $\mathbb{C}$ will denote the fields of real and complex numbers respectively and $\mathbb{R}^{n \times n}\left(\mathbb{C}^{n \times n}\right)$ denotes the space of $n \times n$ matrices with real (complex) entries. For a matrix $A$ in $\mathbb{R}^{n \times n}, A^{T}$ denotes its transpose, $\operatorname{det}(A)$ its determinant and $a_{i j}$ the entry in the $(i, j)$ position of $A$. Similarly for a vector $x$ in $\mathbb{R}^{n}, x_{i}$ denotes the $i^{t h}$ component of $x$. A matrix $A \in \mathbb{R}^{n \times n}$ is said to be symmetric if $A=A^{T}$. The notation $P>0(P \geq 0)$ is used to denote that the matrix $P$ is positive (semi-)definite with $P<0,(P \leq 0)$ meaning that $-P>0$ $(-P \geq 0)$

We shall be concerned with the discrete-time Lyapunov matrix equation ${ }^{1}$

$$
A^{T} P A-P=-Q \quad A, P, Q \in \mathbb{R}^{n \times n}
$$

The next result is standard in the theory of discrete-time LTI systems (Rugh 1996).

\footnotetext{
${ }^{1}$ referred to as the Stein equation by some authors
} 
Theorem 1.1 : Let $A \in \mathbb{R}^{n \times n}$. If there exist $P=P^{T}>0, Q=Q^{T}>0$ satisfying (1), then all of the eigenvalues of the matrix $A$ have magnitude less than 1 . Conversely, if all of the eigenvalues of $A$ have magnitude less than 1 , then there exists a Hermitian solution $P$ to (1) for any choice of Hermitian $Q$ and moreover, if $Q>0$ then $P>0$.

The relevance of equation (1) to the theory of discrete-time LTI systems (and the reason for calling it the discrete-time Lyapunov equation) is provided by the fact (Rugh 1996), (Kailath 1980) that the discrete-time LTI system

$$
\Sigma_{A}: x(k+1)=A x(k) \quad x(0)=x_{0}, A \in \mathbb{R}^{n \times n}
$$

is exponentially stable if there exist positive definite matrices $P, Q$ satisfying (1). We shall refer to such systems as discrete-time stable systems from now on. Note that a system $\Sigma_{A}$ is discrete-time stable if and only if all eigenvalues of the matrix $A$ have magnitude less than 1.

In the spirit of (Shorten, Narendra and Mason 2003), we now define strong and weak CQLFs for a set of discrete-time stable LTI systems.

\section{Strong and weak common quadratic Lyapunov functions}

Consider the set of discrete-time stable LTI systems

$$
\Sigma_{A_{i}}: x(k+1)=A_{i} x(k), i \in\{1,2, \ldots M\}
$$

where the $A_{i}, i \in\{1,2, \ldots M\}$, are constant matrices in $\mathbb{R}^{n \times n}$ such that all the eigenvalues of $A_{i}$ have magnitude less than 1 . Let the matrix $P=P^{T}>0, P \in \mathbb{R}^{n \times n}$, be a simultaneous solution to the discrete-time Lyapunov equations

$$
A_{i}^{T} P A_{i}-P=-Q_{i}, i \in\{1,2, \ldots M\}
$$

If $Q_{i}$ is positive definite, the function $V(x)=x^{T} P x$ is a strong quadratic Lyapunov function for the system $\Sigma_{A_{i}}$. If $Q_{i}$ is positive definite for all $i, 1 \leq i \leq n, V(x)$ is a strong $C Q L F$ for the family of systems $\Sigma_{A_{i}}, 1 \leq i \leq n$. 
Similarly, if $Q_{i}$ is positive semi-definite and singular the function $V(x)=x^{T} P x$ is a weak quadratic Lyapunov function for the system $\Sigma_{A_{i}}$. If $Q_{i}$ is positive semi-definite and singular for all $i, 1 \leq i \leq n, V(x)$ is a weak $C Q L F$ for the family of systems $\Sigma_{A_{i}}, 1 \leq i \leq n$.

The notion of a matrix pencil will be convenient for expressing our later results, so we now recall the definition of this concept.

\section{The matrix pencil $\sigma_{\gamma[0, \infty)}\left[A_{1}, A_{2}\right]$}

The matrix pencil $\sigma_{\gamma[0, \infty)}\left[A_{1}, A_{2}\right]$ is the parameterised family of matrices

$$
\sigma_{\gamma[0, \infty)}\left[A_{1}, A_{2}\right]=\left\{A_{1}+\gamma A_{2}, \gamma \in[0, \infty)\right\}
$$

where $A_{1}, A_{2} \in \mathbb{R}^{n \times n}$. We say that the pencil is $\underline{\text { non-singular }}$ if $A_{1}+\gamma A_{2}$ is non-singular for all $\gamma \geq 0$. Otherwise the pencil is said to be singular.

To complete this section, we recall some facts about the continuous-time Lyapunov equation that will be needed later. A matrix $A \in \mathbb{R}^{n \times n}$ is said to be Hurwitz if all of its eigenvalues lie in the open left half of the complex plane. The following standard result is the continuous-time analogue of Theorem 1.1 (Horn and Johnson 1991), (Rugh 1996).

Theorem 1.2 : Let $A \in \mathbb{R}^{n \times n}$. If there exist positive definite matrices $P$ and $Q$ satisfying the continuous-time Lyapunov equation

$$
A^{T} P+P A=-Q
$$

then $A$ is Hurwitz. Conversely, if $A$ is Hurwitz, then there exists a Hermitian solution $P$ to the equation (6) for any choice of Hermitian matrix $Q$ and moreover, if $Q>0$ then $P>0$.

As in the discrete-time case, equation (6) is intimately related to the stability properties of the continuous-time LTI system

$$
\Sigma_{A}: \frac{d x}{d t}=A x \quad x(0)=x_{0}, A \in \mathbb{R}^{n \times n} .
$$

Using equation (6), the notions of strong and weak CQLFs are defined for continuous-time LTI systems in the obvious way. 


\section{Some Preliminary Lemmas}

In this section, we present some technical results that shall prove useful in establishing the main theorem of the next section. While a number of these results are known, we include them here for the sake of completeness. The following well-known Lemma provides a convenient test for singularity of a matrix pencil.

Lemma 2.1 : Let $A_{1}, A_{2}$ be non-singular matrices in $\mathbb{R}^{n \times n}$. The pencil $\sigma_{\gamma[0, \infty)}\left[A_{1}, A_{2}\right]$ is singular if and only if the matrix product $A_{1} A_{2}^{-1}$ has a negative (real) eigenvalue.

Proof: $A_{1}$ is non-singular so the pencil is not singular for $\gamma=0$. Now if $\gamma>0$ then

$$
\begin{aligned}
\operatorname{det}\left(A_{1}+\gamma A_{2}\right)=0 & \Longleftrightarrow\left(\operatorname{det}\left(A_{1} A_{2}^{-1}+\gamma I\right)\right)\left(\operatorname{det} A_{2}\right)=0 \\
& \Longleftrightarrow \operatorname{det}\left(A_{1} A_{2}^{-1}+\gamma I\right)=0
\end{aligned}
$$

Thus the matrix $A_{1}+\gamma A_{2}$ is singular if and only if the matrix product $A_{1} A_{2}^{-1}$ has the negative (real) eigenvalue $-\gamma$.

We next record the simple observation that the quadratic Lyapunov functions for the discretetime stable LTI systems $\Sigma_{A}$ and $\Sigma_{-A}$ coincide. This relates to the result in (Loewy 1976) identifying the quadratic Lyapunov functions for $\Sigma_{A}$ and $\Sigma_{A^{-1}}$ for continuous-time systems. 2. A related observation was also made in (Barker, Berman and Plemmons 1978).

Lemma 2.2 : $\quad$ Consider the discrete-time stable LTI systems

$$
\begin{aligned}
\Sigma_{A}: x(k+1) & =A x(k) \\
\Sigma_{-A}: x(k+1) & =(-A) x(k) .
\end{aligned}
$$

Then, any quadratic Lyapunov function for $\Sigma_{A}$ is also a quadratic Lyapunov function for $\Sigma_{-A}$

\footnotetext{
${ }^{2}$ In fact, it is shown in (Loewy 1976) that, up to constant factors, $A$ and $A^{-1}$ are the only matrices in $\mathbb{R}^{n \times n}$ sharing the same set of real solutions to the continuous time Lyapunov equation (6)
} 
Proof: This is a straightforward consequence of the fact that

$$
A^{T} P A-P=(-A)^{T} P(-A)-P
$$

for any positive definite matrix $P$.

The following lemma relates the discrete-time Lyapunov equation (1) to the continuous-time Lyapunov equation (6), and introduces a notation that we shall use to state our results.

Lemma 2.3 : Let $\Sigma_{A}$ be a discrete-time stable LTI system, and $P=P^{T}>0$ be a solution of the discrete-time Lyapunov equation

$$
A^{T} P A-P=-Q \quad Q>0 .
$$

Consider the matrix

$$
C(A)=(A-I)(A+I)^{-1}
$$

(Note that this is well defined as the eigenvalues of $A$ are all of modulus less than 1.) Then $P$ is also a solution of the continuous-time Lyapunov equation

$$
C(A)^{T} P+P C(A)=-Q^{\prime}
$$

with $Q^{\prime}=2(A+I)^{-T} Q(A+I)^{-1}>0$ The mapping

$$
A \rightarrow C(A)=(A-I)(A+I)^{-1}
$$

is known as the bilinear transformation in the engineering literature ${ }^{3}$ (Kailath 1980), (Gajic and Qureshi 1995) and it provides a link between the discrete-time and the continuous-time Lyapunov equations. Henceforth, we shall use the notation $C(A)$ for this mapping and write $C^{-1}(A)$ for the inverse mapping $C^{-1}(A)=(I+A)(I-A)^{-1}$.

The next result gives a simple necessary condition for the existence of a strong CQLF for two discrete-time stable LTI systems.

\footnotetext{
${ }^{3}$ It is more commonly referred to as the Cayley transform in mathematics
} 
Lemma 2.4 : Let $\Sigma_{A_{1}}, \Sigma_{A_{2}}$ be two discrete-time stable LTI systems with a strong CQLF given by $V(x)=x^{T} P x$. Then the two matrix pencils $\sigma_{\gamma[0, \infty)}\left[C\left(A_{1}\right), C\left(A_{2}\right)\right], \sigma_{\gamma[0, \infty)}\left[C\left(A_{1}\right), C\left(A_{2}\right)^{-1}\right]$ are non-singular.

Proof: It is straightforward to check that $C(-A)=C(A)^{-1}$. Thus, by Lemma 2.2 and Lemma 2.3, $P$ also defines a strong CQLF for the continuous-time systems $\Sigma_{C\left(A_{1}\right)}, \Sigma_{C\left(A_{2}\right)}$ and for the continuous-time systems $\Sigma_{C\left(A_{1}\right)}, \Sigma_{C\left(A_{2}\right)^{-1}}$. This implies in turn that $P$ defines a strong quadratic Lyapunov function for the systems $\Sigma_{C\left(A_{1}\right)+\gamma C\left(A_{2}\right)}, \Sigma_{C\left(A_{1}\right)+\gamma C\left(A_{2}\right)^{-1}}$ for all $\gamma>0$. From this it follows that the pencils $\sigma_{\gamma[0, \infty)}\left[C\left(A_{1}\right), C\left(A_{2}\right)\right], \sigma_{\gamma[0, \infty)}\left[C\left(A_{1}\right), C\left(A_{2}\right)^{-1}\right]$ must consist entirely of Hurwitz matrices (Theorem 1.2) and, in particular must be nonsingular.

The proofs of the next two results to be presented are a little longer than the others in this section, and for this reason are included in the appendix rather than in the body of the text.

Lemma 2.5 : Let $u, v, x, y \in \mathbb{R}^{n}$ be any four non-zero vectors. There exists a non-singular $T \in \mathbb{R}^{n \times n}$ such that each component of the vectors $T u, T v, T x, T y$ is non-zero.

The next result (Lemma 2.6) establishes a convenient relationship between two parameterizations of the same hyperplane in the space of symmetric matrices in $\mathbb{R}^{n \times n}$. A direct proof of this result is provided in the appendix.

Lemma 2.6 : Let $x, y, u, v$ be 4 non-zero vectors in $\mathbb{R}^{n}$ such that for all $n \times n$ real symmetric matrices $H$

$$
x^{T} H y=-k u^{T} H v
$$

with $k>0$. Then either

$$
x=\alpha u \text { for some real scalar } \alpha, \text { and } y=-\left(\frac{k}{\alpha}\right) v
$$

or

$$
x=\beta v \text { for some real scalar } \beta \text { and } y=-\left(\frac{k}{\beta}\right) u .
$$




\section{Main results}

The principal result of this paper concerns two discrete-time stable LTI systems for which no strong CQLF exists but for which a weak CQLF exists with each of the $Q_{i}, i \in\{1,2\}$ in (4) of rank $n-1$. In Theorem 3.1 we provide a simple algebraic characterisation of this situation. The result is of interest for any class of systems where the transition from the existence of a CQLF to the non-existence of a CQLF passes through the situation described in the theorem.

Remark: It is possible to show that for any matrix $A \in \mathbb{R}^{n \times n}$, all of whose eigenvalues are of modulus less than one, the set of matrices $P=P^{T}$ satisfying

$$
A^{T} P A-P=-Q \quad Q \geq 0, \operatorname{rank}(Q)=n-1
$$

is dense in the set of matrices satisfying

$$
A^{T} P A-A=-Q \quad Q \geq 0, \operatorname{det}(Q)=0
$$

This indicates that the situation described in the theorem is potentially of great importance in providing insight into the existence question for strong and weak CQLFs.

Theorem 3.1 : Let $\Sigma_{A_{1}}, \Sigma_{A_{2}}$ be two discrete-time stable LTI systems such that a positive semi-definite solution $P=P^{T} \geq 0$ exists to the non-strict Lyapunov equations

$$
\begin{aligned}
& A_{1}^{T} P A_{1}-P=-Q_{1} \leq 0, \\
& A_{2}^{T} P A_{2}-P=-Q_{2} \leq 0,
\end{aligned}
$$

for some positive semi-definite matrices $Q_{1}, Q_{2}$ both of rank $n-1(n \geq 2)$. Furthermore suppose that the systems $\Sigma_{A_{1}}, \Sigma_{A_{2}}$ do not have a strong CQLF. Under these conditions, at least one of the pencils $\sigma_{\gamma[0, \infty)}\left[C\left(A_{1}\right), C\left(A_{2}\right)\right], \sigma_{\gamma[0, \infty)}\left[C\left(A_{1}\right), C\left(A_{2}\right)^{-1}\right]$ is singular, and equivalently, at least one of the matrix products $C\left(A_{1}\right) C\left(A_{2}\right)$ and $C\left(A_{1}\right) C\left(A_{2}\right)^{-1}$ has a real negative eigenvalue. 
Proof: As $Q_{1}$ and $Q_{2}$ are of rank $n-1$, there are non-zero vectors $x_{1}, x_{2}$ such that

$$
\begin{aligned}
& x_{1}^{T} Q_{1} x_{1}=0 \\
& x_{2}^{T} Q_{2} x_{2}=0 .
\end{aligned}
$$

The proof of Theorem 3.1 is split into two main stages.

Stage 1 : The first stage in the proof is to show that if there exists a real symmetric matrix $\bar{P}$ satisfying

$$
\begin{aligned}
& x_{1}^{T}\left(A_{1}^{T} \bar{P} A_{1}-\bar{P}\right) x_{1}<0 \\
& x_{2}^{T}\left(A_{2}^{T} \bar{P} A_{2}-\bar{P}\right) x_{2}<0
\end{aligned}
$$

then $\Sigma_{A_{1}}, \Sigma_{A_{2}}$ would have a strong CQLF.

So assume that there is some $\bar{P}$ satisfying (11), (12), and consider the set

$$
\Omega_{1}=\left\{x \in \mathbb{R}^{n}:\|x\|=1 \text { and } x^{T}\left(A_{1}^{T} \bar{P} A_{1}-\bar{P}\right) x \geq 0\right\} .
$$

(Here $\|x\|$ is the usual Euclidean norm on $\mathbb{R}^{n}$.)

We shall show that there is a positive constant $C_{1}>0$ such that $A_{1}^{T}\left(P+\delta_{1} \bar{P}\right) A_{1}-\left(P+\delta_{1} \bar{P}\right)$ is negative definite provided that $0<\delta_{1}<C_{1}$.

Firstly suppose that $\Omega_{1}$ was empty. Then $A_{1}^{T}\left(P+\delta_{1} \bar{P}\right) A_{1}-\left(P+\delta_{1} \bar{P}\right)$ is negative definite for any $\delta_{1}>0$. So any positive constant $C_{1}$ will work in this case.

Now, assume that the set $\Omega_{1}$ is non-empty. The function that takes $x$ to $x^{T}\left(A_{1}^{T} \bar{P} A_{1}-\bar{P}\right) x$ is continuous. Thus $\Omega_{1}$ is closed and bounded, hence compact. Furthermore $x_{1}$ (or any non-zero multiple of $\left.x_{1}\right)$ is not in $\Omega_{1}$ and thus $x^{T}\left(A_{1}^{T} P A_{1}-P\right) x$ is strictly negative on $\Omega_{1}$.

Let $M_{1}$ be the maximum value of $x^{T}\left(A_{1}^{T} \bar{P} A_{1}-\bar{P}\right) x$ on $\Omega_{1}$, and let $M_{2}$ be the maximum value of $x^{T}\left(A_{1}^{T} P A_{1}-P\right) x$ on $\Omega_{1}$. Then by the final remark in the previous paragraph, $M_{2}<0$. 
Choose any constant $\delta_{1}>0$ such that

$$
\delta_{1}<\frac{\left|M_{2}\right|}{M_{1}+1}
$$

and consider the matrix

$$
P+\delta_{1} \bar{P}
$$

By separately considering the cases $x \in \Omega_{1}$ and $x \notin \Omega_{1},\|x\|=1$, it is easy to see that for all non-zero vectors $x$ of norm 1

$$
x^{T}\left(A_{1}^{T}\left(P+\delta_{1} \bar{P}\right) A_{1}-\left(P+\delta_{1} \bar{P}\right)\right) x<0
$$

provided $0<\delta_{1}<\frac{\left|M_{2}\right|}{M_{1}+1}$. Let $C_{1}$ denote the value $\frac{\left|M_{2}\right|}{M_{1}+1}$. Thus we have shown that there is some positive constant $C_{1}$ such that $A_{1}^{T}\left(P+\delta_{1} \bar{P}\right) A_{1}-\left(P+\delta_{1} \bar{P}\right)$ is negative definite provided that $0<\delta_{1}<C_{1}$.

Now the same argument can be used to guarantee the existence of a positive constant $C_{2}$ such that

$$
x^{T}\left(A_{2}^{T}\left(P+\delta_{1} \bar{P}\right) A_{2}-\left(P+\delta_{1} \bar{P}\right)\right) x<0 .
$$

for all non-zero $x$ provided we choose $0<\delta_{1}<C_{2}$. So, if we choose $\delta$ less than the minimum of $C_{1}, C_{2}$, we would have a real symmetric matrix

$$
P_{1}=P+\delta \bar{P}
$$

satisfying (4) with $Q_{1}, Q_{2}>0$. Furthermore it follows from Theorem 1.1 that $P_{1}>0$ and thus $V(x)=x^{T} P_{1} x$ would be a strong CQLF for $\Sigma_{A_{1}}, \Sigma_{A_{2}}$.

Stage 2 : So, under our assumptions there is no real symmetric matrix $H$ such that

$$
\begin{aligned}
& x_{1}^{T}\left(A_{1}^{T} H A_{1}-H\right) x_{1}<0 \\
& x_{2}^{T}\left(A_{2}^{T} H A_{2}-H\right) x_{2}<0 .
\end{aligned}
$$


This means that the mapping from the space of real symmetric matrices in $\mathbb{R}^{n \times n}$ into $\mathbb{R}^{2}$ defined by

$$
H \rightarrow\left(\begin{array}{c}
x_{1}^{T}\left(A_{1}^{T} H A_{1}-H\right) x_{1} \\
x_{2}^{T}\left(A_{2}^{T} H A_{2}-H\right) x_{2}
\end{array}\right)
$$

must be of rank 1 .

Therefore there is some positive constant $k$ such that

$$
x_{1}^{T}\left(A_{1}^{T} H A_{1}-H\right) x_{1}=-k x_{2}^{T}\left(A_{2}^{T} H A_{2}-H\right) x_{2}
$$

for all real symmetric matrices $H$.

Expanding the expression $\left(A_{1} x_{1}-x_{1}\right)^{T} H\left(A_{1} x_{1}+x_{1}\right)$ for symmetric $H$ gives

$$
\begin{aligned}
x_{1}^{T} A_{1}^{T} H A_{1} x_{1}-x_{1}^{T} H x_{1}+x_{1}^{T} A_{1}^{T} H x_{1}-x_{1}^{T} H A_{1} x_{1} & =x_{1}^{T} A_{1}^{T} H A_{1} x_{1}-x_{1}^{T} H x_{1} \\
& =x_{1}^{T}\left(A_{1}^{T} H A_{1}-H\right) x_{1}
\end{aligned}
$$

Therefore

$$
x_{1}^{T}\left(A_{1}^{T} H A_{1}-H\right) x_{1}=\left(A_{1} x_{1}-x_{1}\right)^{T} H\left(A_{1} x_{1}+x_{1}\right)
$$

and similarly

$$
x_{2}^{T}\left(A_{2}^{T} H A_{2}-H\right) x_{2}=\left(A_{2} x_{2}-x_{2}\right)^{T} H\left(A_{2} x_{2}+x_{2}\right)
$$

for all symmetric $H$.

Combining (15) with (16) and (17) yields the following identity for all symmetric matrices $H$.

$$
\left(A_{1} x_{1}-x_{1}\right)^{T} H\left(A_{1} x_{1}+x_{1}\right)=-k\left(A_{2} x_{2}-x_{2}\right)^{T} H\left(A_{2} x_{2}+x_{2}\right)
$$

Now apply Lemma 2.6 to deduce that either

$$
\left(A_{1} x_{1}+x_{1}\right)=\alpha\left(A_{2} x_{2}+x_{2}\right), \quad\left(A_{1} x_{1}-x_{1}\right)=-\frac{k}{\alpha}\left(A_{2} x_{2}-x_{2}\right)
$$


or

$$
\left(A_{1} x_{1}+x_{1}\right)=\alpha\left(A_{2} x_{2}-x_{2}\right), \quad\left(A_{1} x_{1}-x_{1}\right)=-\frac{k}{\alpha}\left(A_{2} x_{2}+x_{2}\right)
$$

In the first case (19), we have

$$
x_{1}=\alpha\left(A_{1}+I\right)^{-1}\left(A_{2}+I\right) x_{2}
$$

and substituting this into the second identity in (19) yields

$$
\left(A_{1}-I\right)\left(A_{1}+I\right)^{-1}\left(A_{2}+I\right) x_{2}=-\frac{k}{\alpha^{2}}\left(A_{2}-I\right) x_{2}
$$

Letting $y=\left(A_{2}+I\right) x_{2}$ we see that

$$
\left(\left(A_{1}-I\right)\left(A_{1}+I\right)^{-1}+\frac{k}{\alpha^{2}}\left(A_{2}-I\right)\left(A_{2}+I\right)^{-1}\right) y=0
$$

and hence the pencil $\sigma_{\gamma[0, \infty)}\left[C\left(A_{1}\right), C\left(A_{2}\right)\right]$ is singular and the product $C\left(A_{1}\right) C\left(A_{2}\right)^{-1}$ has a negative eigenvalue. A similar argument shows that in the case $(20)$, the pencil $\sigma_{\gamma[0, \infty)}\left[C\left(A_{1}\right), C\left(A_{2}\right)^{-1}\right]$ is singular and the product $C\left(A_{1}\right) C\left(A_{2}\right)$ has a negative eigenvalue. This completes the proof of Theorem 3.1.

We note that an alternative proof of Theorem 3.1 is possible by combining the bilinear transformation with results presented in (Shorten, Narendra and Mason 2003).

\section{Comments on the Applicability of the result:}

Theorem 3.1 provides an insight into the general question of CQLF existence for pairs of discrete-time LTI systems and can be used to derive checkable necessary and sufficient conditions for CQLF existence for specific classes of systems of practical importance. The general approach to applying the result relies on the following two facts.

(i) If two systems $\Sigma_{A_{1}}, \Sigma_{A_{2}}$ have a strong CQLF, then the matrix pencils $\sigma_{\gamma[0, \infty)}\left[C\left(A_{1}\right), C\left(A_{2}\right)\right]$, $\sigma_{\gamma[0, \infty)}\left[C\left(A_{1}\right), C\left(A_{2}\right)^{-1}\right]$ are Hurwitz.

(ii) For two systems which do not have a strong CQLF, there is some $d>0$ such that $\Sigma_{A_{1}}$, $\Sigma_{C^{-1}\left(C\left(A_{1}\right)-d I\right)}$ have a weak CQLF but no strong CQLF. 
In order to apply Theorem 3.1, it is necessary to identify systems classes such that the systems obtained in point (ii) above will satisfy the hypotheses of the theorem. To illustrate the method, one such class is presented in the next section. It is the authors belief that other classes exist and the identification of such classes is currently the subject of ongoing research.

It is important to note that for classes to which the result applies in the above sense, it would be possible to derive conditions in terms of the eigenvalue locus of simple matrix pencils which were equivalent to the existence of a strong CQLF. Such conditions are easily checkable and furthermore have a strong connection with the stability properties of the associated switching system. Indeed, the results presented in (Shorten, O'Cairbre and Curran 2000) indicate how such conditions can inform us as to whether or not CQLF existence is a conservative condition for stability. This is of considerable practical importance in using CQLFs to test stability for switching systems.

\section{Second order systems}

In this section we present an example to illustrate the use of Theorem 3.1.

\section{Example: Second order systems}

Let $\Sigma_{A_{1}}$ and $\Sigma_{A_{2}}$ be discrete-time stable LTI systems with $A_{1}, A_{2} \in \mathbb{R}^{2 \times 2}$. We note the following readily verifiable facts.

(a) If a CQLF exists for $\Sigma_{A_{1}}$ and $\Sigma_{A_{2}}$ then the pencils $\sigma_{\gamma[0, \infty)}\left[C\left(A_{1}\right), C\left(A_{2}\right)\right]$ and $\sigma_{\gamma[0, \infty)}\left[C\left(A_{1}\right), C\left(A_{2}\right)^{-1}\right]$ consist entirely of Hurwitz matrices.

(b) If a CQLF does not exist for $\Sigma_{A_{1}}$ and $\Sigma_{A_{2}}$ then a CQLF (in the continuous-time sense) does not exist for the continuous-time systems $\Sigma_{C\left(A_{1}\right)}, \Sigma_{C\left(A_{2}\right)}$. However by choosing $d>0$ sufficiently large, we can ensure that a CQLF (in the continuous-time sense) exists for $\Sigma_{C\left(A_{1}\right)-d I}$ and $\Sigma_{C\left(A_{2}\right)}$. A continuity argument can be employed to show that there is 
some $d_{1}$ with $0<d_{1}<d$ such that $C^{-1}\left(C\left(A_{1}\right)-d_{1} I\right)$ and $A_{2}$ satisfy Theorem 3.1 , and thus one of the pencils $\sigma_{\gamma[0, \infty)}\left[C\left(A_{1}\right)-d_{1} I, C\left(A_{2}\right)\right]$ and $\sigma_{\gamma[0, \infty)}\left[C\left(A_{1}\right)-d_{1} I, C\left(A_{2}\right)^{-1}\right]$ is necessarily singular. Hence, it follows that one of the pencils $\sigma_{\gamma[0, \infty)}\left[C\left(A_{1}\right), C\left(A_{2}\right)\right]$, $\sigma_{\gamma[0, \infty)}\left[C\left(A_{1}\right), C\left(A_{2}\right)^{-1}\right]$ is not Hurwitz.

Items (a) and (b) establish the following facts. Given two discrete-time stable second order LTI systems $\Sigma_{A_{1}}$ and $\Sigma_{A_{2}}$, a necessary condition for the existence of a CQLF is that the pencils $\sigma_{\gamma[0, \infty)}\left[C\left(A_{1}\right), C\left(A_{2}\right)\right]$ and $\sigma_{\gamma[0, \infty)}\left[C\left(A_{1}\right), C\left(A_{2}\right)^{-1}\right]$ are Hurwitz. Conversely,

if a CQLF does not exist for $\Sigma_{A_{1}}, \Sigma_{A_{2}}$, then one of the pencils $\sigma_{\gamma[0, \infty)}\left[C\left(A_{1}\right), C\left(A_{2}\right)\right]$, $\sigma_{\gamma[0, \infty)}\left[C\left(A_{1}\right), C\left(A_{2}\right)^{-1}\right]$ is not Hurwitz. Together these conditions yield the following result which is closely related to that presented in (Akar and Narendra 2001) and is the discrete-time counterpart of results presented in (Shorten and Narendra 1999).

A necessary and sufficient condition for the discrete-time stable LTI systems $\Sigma_{A_{1}}$ and $\Sigma_{A_{2}}, A_{1}, A_{2} \in \mathbb{R}^{2 \times 2}$, to have a $C Q L F$ is that the pencils $\sigma_{\gamma[0, \infty)}\left[C\left(A_{1}\right), C\left(A_{2}\right)\right]$ and $\sigma_{\gamma[0, \infty)}\left[C\left(A_{1}\right), C\left(A_{2}\right)^{-1}\right]$ are Hurwitz.

An example of a real world application where the above result can be applied is the problem of automatic gearbox control. For more information on this topic see the thesis (Shorten 1996).

\section{Concluding remarks}

In this note we have derived a CQLF non-existence theorem. We have applied this theorem to derive a CQLF existence result for a pair of stable LTI systems that belong to a certain system class. We believe that our result can be applied to derive similar results for pairs of stable LTI systems belonging to other important system classes. 


\section{Acknowledgements}

This work was partially supported by the European Union funded research training network Multi-Agent Control, HPRN-CT-1999-00107 ${ }^{4}$ and by the Enterprise Ireland grant SC/2000/084/Y. Neither the European Union or Enterprise Ireland is responsible for any use of data appearing in this publication.

The authors gratefully acknowledge several discussions with Professor Kumpati S. Narendra.

\section{References}

S. Boyd, Q. Yang, Structured and simultaneous Lyapunov functions for system stability problems, Internat. J. Control 49(6):2215-2240 (1989)

R. Loewy, On ranges of real Lyapunov transformations, Lin. Alg. and its Appl. 13:79-89 $(1976)$

T. Ando, Sets of matrices with a common Lyapunov solution, Archiv der Mathematik 77:76-84 $(2001)$

R. N. Shorten, F. O' Cairbre and P. Curran, On the dynamic instability of a class of switching systems, Proceedings of the IFAC conference on Artificial Intelligence in Real Time Control (2000)

R. N. Shorten, A Study of hybrid dynamical systems with application to automotive control, PhD Thesis, Dept. of Electrical and Electronic Engineering, University College Dublin (1996)

R. DeCarlo, M. Branicky, S. Pettersson, B. Lennartson, Perspectives and results on the stability and stabilizability of hybrid systems, IEEE Proceedings 88(7):1069-1082 (2000)

\footnotetext{
${ }^{4}$ This work is the sole responsibility of the authors and does not reflect the European Union's opinion
} 
M. Branicky, Stability of switched and hybrid systems, Proc. IEEE Conference on Decision and Control 3498-3503 (1994)

H. Ye, A. N. Michel, L. Hou, Stability Theory for Hybrid Dynamical Systems, IEEE Transactions on Automatic Control 43(4):461-474 (1998)

K. S. Narendra, J. Balakrishnan, A common Lyapunov function for stable LTI systems with commuting A-matrices, IEEE Transactions on Automatic Control 39(12):1669-1686 (1994)

K. S. Narendra, J. Balakrishnan, Improving transient response of adaptive control systems using multiple models and switching, IEEE Trans. Aut. Control 39:1861-1866 (1994)

R. N. Shorten, F. O'Cairbre, A new methodology for the stability analysis of pairwise triangularisable and related switching systems, Institute of Mathematics and its Applications, Journal of Applied Mathematics, In press (2002)

F. O'Cairbre, R. N. Shorten, A proof of global attractivity for a class of switching systems using a non-quadratic Lyapunov approach, IMA Journal of Mathematical Control and Information 18:341-353 (2001)

R. N. Shorten, K. S. Narendra, On the stability and existence of common Lyapunov functions for stable linear switching systems, Proceedings of the 36th IEEE Conference on Decision and Control $3723-3724$ (2000)

R. N. Shorten, K. S. Narendra, Investigating the stability of a class of hybrid system, IEE Computing and Control Engineering Journal 9(2):81-88 (1998)

A. A. Agrachev, D. Liberzon, Lie-algebraic stability criteria for switched systems, SIAM Journal of Control and Optimization 40(1):253-269 (2001)

G. P. Barker, A. Berman, R. J. Plemmons, Positive diagonal solutions to the Lyapunov equations, Linear and Multilinear Algebra 5:249-256 (1978) 
R. N. Shorten, K. S. Narendra, O. Mason, A result on common quadratic Lyapunov functions, IEEE Transactions on Automatic Control 48(1):110-113 (2003)

L. Gurvits, Stability of discrete linear inclusion, Lin. Alg. and its Appl. 231:47-85 (1995)

R. N. Shorten, K. S. Narendra, Necessary and sufficient conditions for the existence of a common quadratic Lyapunov function in two stable second order linear systems, in: Proceedings of American Control Conference (1999)

M. Johansson, A. Rantzer, Computation of piecewise quadratic Lyapunov functions for hybrid systems, IEEE Transactions on Automatic Control - Special Issue, Hybrid Systems $43: 555-559(1998)$

T. Ooba, Y. Funahashi, On the simultaneous diagonal stability of linear discrete-time systems, Systems \& Control Letters 36:175-180 (1999)

T. Ooba, Y. Funahashi, Two conditions concerning common quadratic Lyapunov functions for linear systems, IEEE Transactions on Automatic Control 42(5):719-721 (1997)

Y. Mori, T. Mori, Y. Kuroe, A set of discrete-time linear systems which have a common quadratic Lyapunov function and its extension, Proceedings of the American Control Conference 2905-2906 (1998)

Y. Mori, T. Mori, Y. Kuroe, Some new subclasses of systems having a common quadratic Lyapunov function and comparison of known subclasses, Proceedings of 40th IEEE Conference on Decision and Control 2179-2180 (2001)

M. Akar, K. S. Narendra, On the existence of a common quadratic Lyapunov function for two stable second order LTI discrete-time systems, in: Proceedings of American Control Conference (2001)

R. A. Horn, C. R. Johnson, Topics in Marix Analysis, Cambridge University Press, Cambridge, 1991 
W. J. Rugh, Linear System Theory (Second Edition), Prentice-Hall information and system science series, Prentice-Hall, Englewood Cliffs, N.J., 1996

Z. Gajic, M. Qureshi, Lyapunov Matrix Equation in System Stability and Control, Mathematics in Science and Engineering vol. 195, Academic Press, 1995

T. Kailath, Linear Systems, Prentice-Hall, Englewood Cliffs, N.J., 1980

\section{Appendix}

\section{Proof of Lemma 2.5}

Consider the norm $\|A\|_{\infty}=\sup \left\{\left|a_{i j}\right|: 1 \leq i, j \leq n\right\}$ on $\mathbb{R}^{n \times n}$, and let $z$ be any non-zero vector in $\mathbb{R}^{n}$. Then it is easy to see that the set $\left\{T \in \mathbb{R}^{n \times n}: \operatorname{det}(T) \neq 0,(T z)_{i} \neq 0,1 \leq i \leq n\right\}$ is open. On the other hand, if $T \in \mathbb{R}^{n \times n}$ is such that $(T z)_{i}=0$ for some $i$, an arbitrarily small change in an appropriate element of the $i^{\text {th }}$ row of $T$ will result in a matrix $T^{\prime}$ such that $\left(T^{\prime} z\right)_{i} \neq 0$. From this it follows that arbitrarily close to the original matrix $T$, there is some $T_{1} \in \mathbb{R}^{n \times n}$ such that $T_{1} z$ is non-zero component-wise.

Now to prove the lemma, simply select a non-singular $T_{0}$ such that $T_{0} x$ is non-zero componentwise. Suppose that some component of $T_{0} y$ is zero. By the arguments in the previous paragraph, it is clear that we can select a non-singular $T_{1} \in \mathbb{R}^{n \times n}$ such that each component of $T_{1} x$ and $T_{1} y$ is non-zero. Now it is simply a matter of repeating this step for the remaining vectors $u$ and $v$ to complete the proof of the lemma.

\section{Proof of Lemma 2.6}

We can assume that all components of $x, y, u, v$ are non-zero. To see why this is so, suppose that the result was proven for this case and we were given four arbitrary non-zero vectors $x, y, u, v$. We could transform them via a single non-singular transformation $T$ such that each component of $T x, T y, T u, T v$ was non-zero (Lemma 2.5). Then for all symmetric matrices $P$ 
we would have

$$
\begin{aligned}
(T x)^{T} P(T y) & =x^{T}\left(T^{T} P T\right) y \\
& =-k u^{T}\left(T^{T} P T\right) v \\
& =-k(T u)^{T} P(T v)
\end{aligned}
$$

Then $T x=\alpha T u$ and thus $x=\alpha u$ or $T x=\beta T v$ and $x=\beta v$. So we shall assume that all components of $x, y, u, v$ are non-zero. We write $x_{i}$ for the $i^{t h}$ component of the vector $x$ and $p_{i j}$ denotes the entry in the $(i, j)$ position of the matrix $P$.

Suppose that $x$ is not a scalar multiple of $u$ to begin with. Then for any index $i$ with $1 \leq i \leq n$, there is some other index $j$ and two non-zero real numbers $c_{i}, c_{j}$ such that

$$
x_{i}=c_{i} u_{i}, x_{j}=c_{j} u_{j}, c_{i} \neq c_{j}
$$

Choose one such pair of indices $i, j$. Equating the coefficients of $p_{i i}, p_{j j}$ and $p_{i j}$ respectively in the identity $x^{T} P y=-k u^{T} P v$ yields the following equations.

$$
\begin{gathered}
x_{i} y_{i}=-k u_{i} v_{i} \\
x_{j} y_{j}=-k u_{j} v_{j} \\
\left(x_{i} y_{j}+x_{j} y_{i}\right)=-k\left(u_{i} v_{j}+u_{j} v_{i}\right)
\end{gathered}
$$

If we combine (21) with (22) and (23), we find

$$
\begin{aligned}
& y_{i}=-\frac{k}{c_{i}} v_{i} \\
& y_{j}=-\frac{k}{c_{j}} v_{j}
\end{aligned}
$$


Using (25), (26), (22), (23) and (24) we find

$$
\begin{aligned}
c_{i} u_{i} y_{j}+c_{j} u_{j} y_{i} & =-k\left(u_{i} v_{j}+u_{j} v_{i}\right) \\
\Rightarrow-\frac{c_{i} k}{c_{j}} u_{i} v_{j}-\frac{c_{j} k}{c_{i}} u_{j} v_{i} & =-k\left(u_{i} v_{j}+u_{j} v_{i}\right) \\
\Rightarrow u_{i} v_{j}\left(\frac{c_{j}-c_{i}}{c_{j}}\right) & =u_{j} v_{i}\left(\frac{c_{j}-c_{i}}{c_{i}}\right)
\end{aligned}
$$

Recall that $c_{i} \neq c_{j}$ so we can divide by $c_{j}-c_{i}$ and rearrange terms to get

$$
\frac{c_{i}}{c_{j}}=\left(\frac{v_{i}}{v_{j}}\right)\left(\frac{u_{j}}{u_{i}}\right)
$$

But using (21) we find

$$
\frac{c_{i}}{c_{j}}=\left(\frac{x_{i}}{x_{j}}\right)\left(\frac{u_{j}}{u_{i}}\right)
$$

Combining (27) and (28) yields

$$
\frac{v_{i}}{v_{j}}=\frac{x_{i}}{x_{j}}
$$

Thus $x_{i}=c v_{i}, x_{j}=c v_{j}$ for some constant $c$.

Now if we select any other index $k$ with $1 \leq k \leq n$, and write

$$
x_{k}=c_{k} u_{k}
$$

then $c_{k}$ must be different to at least one of $c_{i}, c_{j}$. Without loss of generality, we may take it that $c_{k} \neq c_{i}$. Then the above argument can be repeated with the indices $i$ and $k$ in place of $i$ and $j$ to yield

$$
x_{i}=c v_{i}, x_{k}=c v_{k} .
$$

But this can be done for any index $k$ so we conclude that $x=c v$ for a scalar $c$. So we have shown that if $x$ is not a scalar multiple of $u$, then it is a scalar multiple of $v$.

To complete the proof, note that if $x=\beta v$ for a scalar $\beta$ then by (22),

$$
\beta v_{i} y_{i}=-k u_{i} v_{i}
$$

for all $i$. Thus $y=-\left(\frac{k}{\beta}\right) u$ as claimed. The same argument will show that if $x=\alpha u$ for a scalar $\alpha$, then $y=-\left(\frac{k}{\alpha}\right) v$. 